\title{
Practical Modelling for Generating Self-similar VBR Video Traffic
}

\author{
Jong-Suk R. Lee ${ }^{1}$ and Hae-Duck J. Jeong ${ }^{2}$ \\ ${ }^{1}$ Grid Technology Research Department, Supercomputing Centre, \\ Korea Institute of Science and Technology Information, \\ Daejeon, South Korea \\ jsruthlee@kisti.re.kr \\ 2 Department of Information Science, Korean Bible University, \\ Seoul, South Korea \\ joshua@bible.ac.kr
}

\begin{abstract}
Teletraffic in the Internet is rapidly growing and diversifying, and there is a strong need for QoS support in high-speed communication networks. There are a number of research issues concerning the transmission of JPEG/ MPEG video over modern high speed computer networks. These problems have been studied intensively over the last ten years in order to provide a consistent and desirable QoS for JPEG/MPEG video traffic, construct accurate models for JPEG/MPEG video traffic and utilise efficient resource allocation techniques. In the paper we show that synthetically generated streams of VBR video, compressed under such standards as JPEG, MPEG-1 and MPEG-2, can be statistically equivalent to real video traces. We also investigate how compression algorithms on correlation structure of compressed teletraffic influence real video traffic.
\end{abstract}

\section{Introduction}

Teletraffic in the Internet is rapidly growing and diversifying, and there is a strong need for QoS (Quality of Service) support in high-speed communication networks [1], 6]. The introduction of many new multimedia services requires a high bandwidth to transport data such as real-time digital video. Modern computer networks can no longer cope with uncompressed multimedia traffic, resulting in the development of several image and video compression standards such as JPEG and MPEG. In this paper we focus on VBR (Variable Bit Rate) JPEG/MPEG video, i.e., on video streams compressed according to JPEG and MPEG standards and transmitted as VBR components of an ATM network.

There are a number of research issues concerning the transmission of JPEG/ MPEG video over modern high speed computer networks, such as the dimensioning of multiplexer buffers and monitoring of video cell streams. These problems have been studied intensively over the last ten years in order to provide a consistent and desirable QoS for JPEG/MPEG video traffic, construct accurate models for JPEG/MPEG video traffic and utilise efficient resource allocation 
techniques. We look at the influence of compression algorithms on correlation structure of compressed teletraffic, see Section 4, where results of compression of Star Wars video under JPEG and MPEG-1 are discussed.

Applicability of gamma/Pareto model as marginal distributions of compressed video streams is discussed in Section 4, where we look at accuracy of this approximation in relation to data coming from different videos (Star Wars and Titanic) compressed under three different algorithms (JPEG, MPEG-1 and MPEG-2). We also show that synthetically generated streams of VBR video, compressed under such standards as JPEG, MPEG-1 and MPEG-2, are statistically similar to real video traces [7].

A number of researchers tried to fit a specific mathematical model to traces of real VBR video traffic. For example, several models (based on gamma 3], lognormal 10, and combined gamma/Pareto 2, 9]) have been suggested for VBR video traffic. Heyman et al. 3 used a 30-minute compressed videoteleconferencing sequence for simulation studies using the gamma model. Krunz et al. [10] used a 23-minute movie, The Wizard of $\mathrm{Oz}$, to study statistical characteristics of VBR MPEG-coded video streams using the lognormal model. The gamma model for video traffic became inaccurate in the tail of distribution, and the lognormal model was too heavy-tailed at first and then fell off too rapidly. Garrett and Willinger [2] used a two-hour VBR video, Star Wars, and proposed a hybrid gamma/Pareto model based on the F-ARIMA process 4. They found that the tail behaviour of the marginal distribution can be accurately described using the heavy-tailed Pareto distributions.

Huang et al. 5 presented a unified approach to modelling VBR video traffic using both SRD (Short-Range Dependent) and LRD (Long-Range Dependent) empirical ACFs (Auto-correlation Functions). They applied this approach to 2 hours' trace of Last Action Hero video. Their approach is potentially accurate, but establishing an automatic search for the best background ACF remains an open problem. Lombardo et al. 12] proposed the generation of pseudo-MPEG video traffic with a specific correlation structure based on FFT [14 and an ICDF transformation, assuming an arbitrary marginal distribution of the output process. The proposed algorithm has been used to generate a sequence with the same statistical characteristics as those of the movie "The Simpsons", however, the robustness of this algorithm remains an issue open to further investigation.

\section{JPEG/MPEG Video Compression}

Several algorithms have been developed to compress video data, in order to reduce the memory required for their storage, the time or bandwidth necessary for their transmission, and the effective data access or transfer rate. We focus on MPEG-1 and MPEG-2 of the MPEG standard family. MPEG-2 uses encoders from the MPEG-1 scheme, and in the case of multi-layer encoding, the statistical properties of its base layer are almost identical to MPEG-1. A video sequence is simply a series of pictures taken at closely spaced time intervals starting with a sequence header. The sequence header is followed by one or more group(s) of 
Table 1. Parameters for generating the Titanic video sequence

\begin{tabular}{|l|c|}
\hline Parameters & Values \\
\hline \hline Coding algorithm & DCT \\
\hline Duration & 3 hours \\
\hline Video frames & 285,890 (I-, P- and B-frames only) \\
\hline Frame dimensions & 720 x 576 pixels \\
\hline Pixel resolution & 24 bits $/$ pixel (colour) \\
\hline Frame rate & $29.97 /$ second \\
\hline Average bandwidth & 692,150 bytes $/$ second \\
\hline Average compression rate & 53.87 \\
\hline A group of pictures & 15 frames (IBBPBBPBBPBBPBB) \\
\hline
\end{tabular}

pictures (GOP) and ends with a sequence end code. Additional sequence headers may appear between any GOP within the video sequence. This is achieved by using three types of frames: Intra-coded picture (I-frame), Predictive-coded picture (P-frame) and Bi-directionally predictive-coded picture (B-frame) 13 .

Only I- and P-frames can be used as a reference for past and/or future prediction. An ordered collection of I-, P- and B-frames is called a group of pictures. The proportion of I-, P- and B-frames is application-dependent and is left to the user. For example, for many scenes, spacing the reference frames at about onetwelfth of a second interval seems appropriate, i.e., IBBPBBPBBPBB... The MPEG GOP pattern was used to encode the MPEG-1 version of Star Wars by Garrett and Willinger [2].

We encoded three hours of Titanic video to obtain a realistic full-length trace of video traffic. This will be used as a control reference self-similar trace in our investigations. Parameters of the sequence are summarised in Table 1. We chose MPEG-2 to obtain encoded frame sequences of our trace. In this paper we will use the following three self-similar sequences: (i) two hours of Star Wars video encoded by JPEG [2, (ii) two hours of Star Wars video encoded by MPEG-1 2], and (iii) three hours of Titanic video encoded by MPEG-2 [7. The last trace was obtained by taking a sample that was approximately $60 \%$ longer than Sequence (i) and (ii).

\section{$3 \quad$ Modelling for Self-similar VBR Video Traffic}

Following the recommendation of Garrett and Willinger [2, we chose to use the combined gamma/Pareto model for VBR video traffic. They along with Krunz and Makowski [9], showed that the gamma distribution can be used to capture the main part of the empirical distribution, but is inappropriate for the tail. Addition of a heavy-tailed Pareto distribution corrects this, as shown in Figure 1].

Let $F_{\Gamma}$ and $F_{P}$ be the CDF (Cumulative Distribution Function) for the gamma and Pareto distributions, respectively. Note that $F_{\Gamma}$ has no closed form 


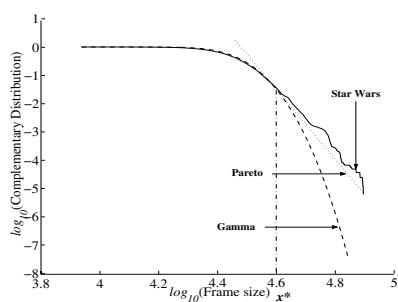

(a) Star Wars JPEG

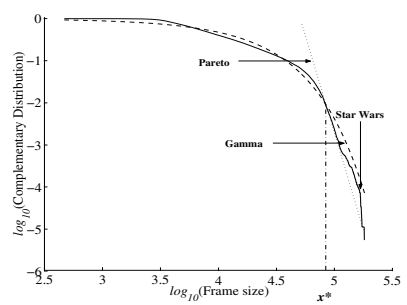

(b) Star Wars MPEG-1

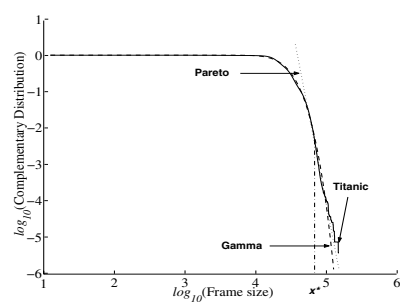

(c) Titanic MPEG-2

Fig. 1. Complementary cumulative distributions of real video traffic and gamma/Pareto model

of the CDF when $\alpha_{\Gamma}$, the shape parameter of the gamma distribution assumes non-integer values.

If $\alpha_{\Gamma}$ is a positive integer, then the CDF for the gamma distribution is given by

$$
F_{\Gamma}(x)= \begin{cases}0, & \text { for } x \leq 0, \\ 1-e^{-x / \beta_{\Gamma}} \sum_{j=0}^{\alpha_{\Gamma}-1} \frac{\left(x / \beta_{\Gamma}\right)^{j}}{j !}, & \text { for } x>0,\end{cases}
$$

where $\alpha_{\Gamma}$ is the shape parameter, $\alpha_{\Gamma}>0$, and $\beta_{\Gamma}$ is the scale parameter, $\beta_{\Gamma}>0$.

The $\mathrm{CDF} F_{P}(x)$ of the Pareto distribution is given as:

$$
F_{P}(x)= \begin{cases}0, & \text { for } x<1, \\ 1-\left(\frac{b_{P}}{x}\right)^{\alpha_{P}}, & \text { for } 1 \leq x \leq \infty,\end{cases}
$$

where $\alpha_{P}$ is the shape parameter, $\alpha_{P}>0$, and $b_{P}$ is the minimum allowed value of $x, 0<b_{P} \leq x$.

Thus, the combined gamma/Pareto distribution is determined by

$$
F_{\Gamma / P}(x)=\left\{\begin{array}{l}
0, \quad \text { for } x \leq 0, \\
F_{\Gamma}(x), \text { for } 0<x \leq x^{*}, \\
F_{P}(x), \text { for } x>x^{*}
\end{array}\right.
$$

The complementary CDFs of $F_{\Gamma}(x)$ and $F_{P}(x)$ can be used to determine $x^{*}$ in Equation (3). The parameters of the gamma distribution are obtained by matching the first and second moments of the empirical sequence to those of a gamma random variate. $x^{*}$ can be obtained graphically by inspecting the tail behaviour of the empirical distribution, and determining where it starts to deviate from the tail of the gamma curve. The values of $b_{P}$ and $\alpha_{P}$ for the estimated Pareto distribution can be obtained by finding $x=x^{*}$ for which the least-square fit of the Pareto tail gives $F_{\Gamma}(x)=F_{P}(x)$. Figure 1 shows log-log plots of gamma and Pareto complementary CDF for real VBR video traffic. While the gamma curve fits the main part of the empirical video traffic well, the Pareto curve closely fits its tail part. Applying this method, we have determined values of $x^{*}$ for all three samples; see Table 2 . 
Table 2. Estimated parameter values obtained from Star Wars and Titanic video traffic utilising the combined gamma/Pareto model

\begin{tabular}{|l|r|r|r|}
\hline Parameters & \multicolumn{3}{|c|}{ Estimated values } \\
\cline { 2 - 4 } & Star Wars JPEG & Star Wars MPEG-1 & Titanic MPEG-2 \\
\hline \hline Length (frames) & 171,000 & 174,136 & 285,890 \\
\hline Duration & 2 hours & 2 hours & 3 hours \\
\hline $\begin{array}{l}\text { Compression } \\
\text { algorithm }\end{array}$ & Intra-frame & MPEG-1 & MPEG-2 \\
\hline Sample mean & 27,791 & 15,598 & 26,353 \\
\hline Standard dev. & 6,254 & 18,165 & 11,600 \\
\hline Maximum & 78,459 & 185,267 & 146,608 \\
\hline Minimum & 8,622 & 476 & 12 \\
\hline Gamma $\alpha_{\Gamma}$ & 25.8 & 0.737 & 5.16 \\
\hline Gamma $\beta_{\Gamma}$ & 1,100 & 21,154 & 5,106 \\
\hline Pareto $\alpha_{P}$ & 12.42 & 9.19 & 10.06 \\
\hline Pareto $b_{P}$ & 30,000 & 51,500 & 37,800 \\
\hline$x^{*}$ & 39,810 & 86,003 & 57,280 \\
\hline
\end{tabular}

Given a self-similar sequence of the FGN-DW (Fractional Gaussian NoiseDaubechies Wavelets) process $\mathbf{X}[8$, we can transform the marginal distribution by mapping each point as

$$
Z_{i}=F_{\Gamma / P}^{-1}\left(F_{N}\left(X_{i}\right)\right), \quad i=1,2, \ldots,
$$

where $F_{N}(\cdot)$ is the $\mathrm{CDF}$ of the normal distribution and $F_{\Gamma / P}^{-1}(\cdot)$ is the inverse $\mathrm{CDF}$ of the combined gamma/Pareto model given by

$$
F_{\Gamma / P}^{-1}(y)=\left\{\begin{array}{cl}
F_{\Gamma}^{-1}(y), & \text { for } y \leq 1-\left(b_{P} / x^{*}\right)^{\alpha_{P}}, \\
F_{P}^{-1}(y)=b_{P} /(1-y)^{1 / \alpha_{P}}, & \text { for } y>1-\left(b_{P} / x^{*}\right)^{\alpha_{P}} .
\end{array}\right.
$$

Note that for computing $F_{\Gamma}^{-1}(y)$, we used the Newton-Raphson technique [1], 15. The procedure for the MPEG video consists of I-, P- and B-frames of sequences generated from FGN-DW [8], which are then combined in I-, B- and P-frame order (e.g., IBBPBBPBBPBBPBBI ...) before transforming the corresponding time series into time series with the gamma/Pareto marginal distributions defined in Equation (44). The procedure for the JPEG synthetic sequence generated from FGN-DW is simple. We used the sequences obtained from the previous procedure for simulation studies of VBR video traffic, which we describe in the next section.

\section{$4 \quad$ Numerical Results}

\subsection{Analysis of Hurst Parameter Estimates for VBR Video Traffic}

The Hurst parameter estimates obtained from the most efficient estimators (i.e., the wavelet-based $H$ estimator and Whittle's MLE) [7, have been used to anal- 
Table 3. Estimates of the Hurst parameter obtained from the wavelet-based $H$ estimator and Whittle's MLE for Star Wars JPEG, Star Wars MPEG-1 and Titanic MPEG-2 video traffic. We give $95 \%$ confidence intervals for the means of two estimators in parentheses

\begin{tabular}{|l|c|c|c|}
\hline Estimators & Star Wars JPEG & Star Wars MPEG-1 & Titanic MPEG-2 \\
\hline \hline Wavelet-based & $.8841(.879, .889)$ & $.8634(.859, .868)$ & $.9034(.895, .911)$ \\
\hline Whittle's MLE & $.8997(.887, .912)$ & $.8680(.855, .880)$ & $.8999(.886, .914)$ \\
\hline
\end{tabular}

yse Star Wars JPEG, Star Wars MPEG-1 and Titanic MPEG-2 video sequences. Table 3 shows the estimates of the Hurst parameter for the three video sequences. Comparing Star Wars after JPEG and MPEG-1, we can formulate hypothesis that JPEG produces stronger dependent output video sequences. Our results show $2 \%$ difference in $H$ parameter when using the wavelet-based $H$ estimator, and $11 \%$ difference when using Whittle's MLE, see Table 3. On the other hand, two different videos (Star Wars and Titanic) show that regardless of compression algorithm resulted processes have the same marginal distribution well approximated by our gamma/Pareto model. This can be regarded as generalisation of a finding by Garrett and Willinger 2] who showed that gamma/Pareto model is a good approximation of marginal distributions for Star Wars compressed under JPEG.

The wavelet-based $H$ estimator of three hours of real Titanic video traffic calculates $\hat{H}=0.9034$, shown in Table 3 . Estimate of the Hurst parameter $\hat{H}$ obtained from Whittle's MLE is 0.8999. The Hurst parameter estimates for the Star Wars JPEG and Star Wars MPEG-1 video sequences are also given in Table 3.

\subsection{Simulation Results of the VBR Video Traffic Model}

Figure 2 shows quantile-quantile plots for the distribution of frame sizes in bytes of real VBR video traffic, (i.e., Star Wars JPEG, Star Wars MPEG-1, and Titanic MPEG-2), and the combined gamma/Pareto model based on FGN-DW. We observed that although the compression algorithms used for encoding the various videos were different, the combined model fits the real traffic statistic well. Note especially that the distribution of the gamma/Pareto model matches the real Star Wars JPEG video traffic well.

Figure 3 shows that the ACF of the combined gamma/Pareto model also fit the empirical video traffic statistic well. The ACF curve of the gamma/Pareto model at large lags (i.e., lags $>1,800$ ) fit the real Star Wars JPEG video traffic well, but the model slightly underestimated at small lags. The ACF curves in Figures 3 (b) and (c) also oscillated more than the one in Figure 3 (a), due to the MPEG format. Furthermore, the autocorrelation structure in Figure 3 (b) oscillated more than that in Figure 3 (c) because they use different frame formats (i.e., while every 12th frame in Figure 3 (b) is an I-frame, every 15th frame in Figure 3 (c) is an I-frame). In addition, we found strong evidence of LRD, as all ACF curves obtained from the real video traffic and the gamma/Pareto model 


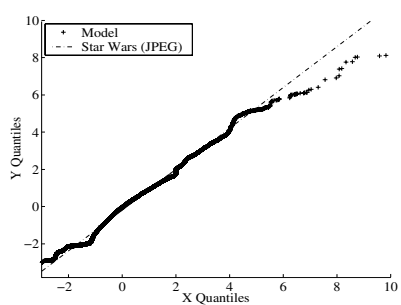

(a) Star Wars JPEG

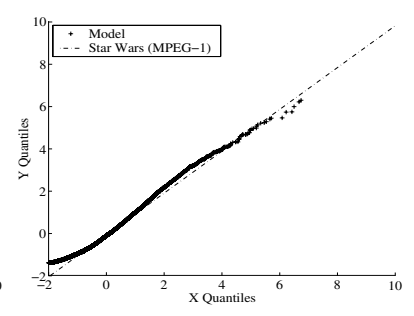

(b) Star Wars MPEG-1

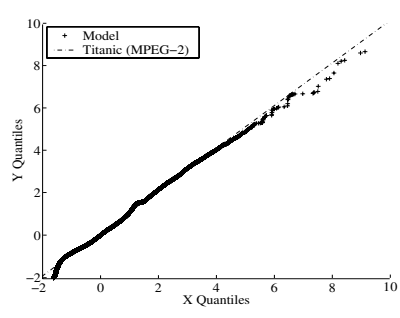

(c) Titanic MPEG-2

Fig. 2. Distributions of real video traffic and traffic from the gamma/Pareto models

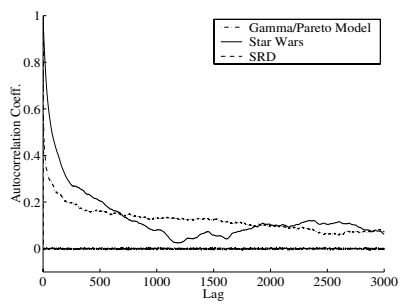

(a) Star Wars JPEG

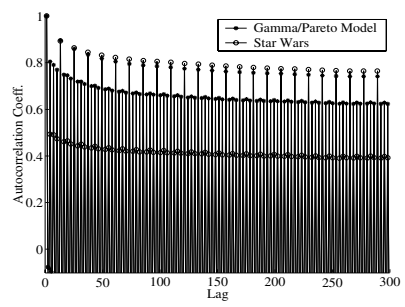

(b) Star Wars MPEG-1

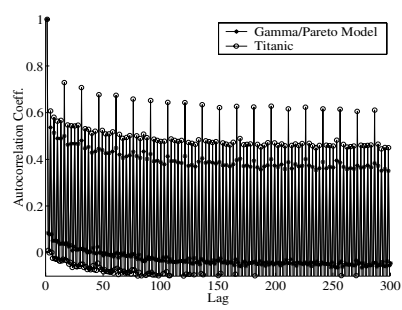

(c) Titanic MPEG-2

Fig. 3. ACF of real video traffic and traffic from the gamma/Pareto models

decayed slowly, while the SRD (i.e., Poisson model) in Figure 3 (a) decayed quickly.

\section{Conclusions}

We showed how pseudo-random self-similar sequences can be applied to produce a model of teletraffic associated with the transmission of VBR JPEG/MPEG video. A combined gamma/Pareto model based on the application of the FGNDW generator was used to synthesise VBR JPEG/MPEG video traffic.

In the paper we showed that synthetically generated streams of VBR video, compressed under such standards as JPEG, MPEG-1 and MPEG-2, can be statistically equivalent to real video traces shown in Section 4 (see also Figures 2 - 3). We investigated how compression algorithms on correlation structure of compressed teletraffic influence real video traffic. Generalisation of findings of Garrett and Willinger, showing that video compression algorithms (MPEG-1 and MPEG-2) lead to self-similar processes was studied. We considered outcomes of MPEG-1 and MPEG-2 in addition to previously studied outcomes of JPEG [2], to show that the results of (Garrett and Willinger [2]) do not depend on the compression algorithms. 
Better GOP and cell-layered modelling is needed for development of integrated MPEG video traffic models. While some general assessment of queueing performance can be obtained from single-streams, more universal results could be obtained from the queueing performance analysis of multiplexed streams of video traffic. These issues await further investigations.

\section{Acknowledgements}

The authors thank Dr. Don McNickle, Dr. Krzysztof Pawlikowski, Dr. Manfred Jobmann, Dr. Matthew Roughan and three anonymous referees for their valuable comments. The authors also wish to thank the financial support of Korean Bible University, and Korea Institute of Science and Technology Information, Korea.

\section{References}

1. Fahmy, S., Jain, R., Rabie, S., Goyal, R., And Vandalore, B. Quality of Service for Internet Traffic over ATM Service Categories. Computer Communications 22, 14 (1999), 1307-1320.

2. Garrett, M., And Willinger, W. Analysis, Modeling and Generation of SelfSimilar VBR Video Traffic. In Computer Communication Review, Proceedings of ACM SIGCOMM'94 (London, UK, 1994), vol. 24 (4), pp. 269-280.

3. Heyman, D., Tabatabai, A., and Lakshman, T. Statistical Analysis and Simulation Study of Video Teleconference Traffic in ATM. IEEE Transactions on Circuits and Systems for Video Technology 2, 1 (1992), 49-59.

4. Hosking, J. M. Fractional Differencing. Biometrika 68, 1 (1981), 165-176.

5. Huang, C., Devetsikiotis, M., Lambadaris, I., and Kaye, A. Modeling and Simulation of Self-Similar Variable Bit Rate Compressed Video: A Unified Approach. Computer Communication Review, Proceedings of ACM SIGCOMM'95 25, 4 (1995), 114-125.

6. JAIn, R. Current Issues in Telecom Networks: QoS, Traffic Engineering and DWDM. Keynote speaker, http://www.cis.ohio-state.edu/ jain/ talks/icon99.htm, 2000.

7. Jeong, H.-D. J. Modelling of Self-Similar Teletraffic for Simulation. PhD thesis, Department of Computer Science, University of Canterbury, 2002.

8. JeOng, H.-D. J., McNickle, D., And Pawlikowski, K. Fast Self-Similar Teletraffic Generation Based on FGN and Wavelets. In Proceedings of the IEEE International Conference on Networks, ICON'99 (Brisbane, Australia, 1999), pp. 75-82.

9. Krunz, M., And Makowski, A. A Source Model for VBR Video Traffic Based on $M / G / \infty$ Input Processes. In Proceedings of IEEE INFOCOM'98 (San Francisco, CA, USA, 1998), pp. 1441-1448.

10. Krunz, M., Sass, R., And Hughes, H. Statistical Characteristics and Multiplexing of MPEG Streams. In Proceedings of IEEE INFOCOM'95 (Boston, Massachusetts, 1995), pp. 455-462.

11. Law, A., And Kelton, W. Simulation Modeling and Analysis. 2nd ed., McGrawHill, Inc., Singapore, 1991.

12. Lombardo, A., Morabito, G., Palazzo, S., and Schembra, G. MPEG Traffic Generation Matching Intra- and Inter-GoP Correlation. Simulation 74, 2 (2000), 97-109. 
13. Mitchell, J., Pennebaker, W., Fogg, C., And LeGall, D. MPEG Video Compression Standard. Chapman and Hall, New York, 1997.

14. Paxson, V. Fast, Approximate Synthesis of Fractional Gaussian Noise for Generating Self-Similar Network Traffic. Computer Communication Review, ACM SIGCOMM 27, 5 (1997), 5-18.

15. Press, W., Teukolsky, S., Vetterling, W., and Flannery, B. Numerical Recipes in C. Cambridge University Press, Cambridge, 1999. 